\title{
Motion Center in Quantum Wave Packet Dynamics
}

\author{
QIAN LiU ${ }^{a, b}$, YANXIA YU ${ }^{c}$, HUi PAN ${ }^{d, *}$ AND Z.S. WANG ${ }^{a, b, *}$ \\ ${ }^{a}$ College of Physics and Communication Electronics, Jiangxi Normal University, Nanchang 330022, P.R. China \\ ${ }^{b}$ Center for Quantum Science and Technology, Nanchang 330022, P.R. China \\ ${ }^{c}$ School of Physical Science and Technology, Wuhan University, Wuhan 430072, P.R. China \\ ${ }^{d}$ Institute of Applied Physics and Materials Engineering, Faculty of Science and Technology, \\ University of Macau, Macao SAR, China
}

(Received September 20, 2013; revised version March 25, 2014; in final form December 10, 2014)

We seek for an oscillating center solution of the wave function satisfying the Schrödinger equation for a nonrelativistic charge particle in an arbitrary external field, where the oscillating center of physical system is a motion governing by a guidance formula of the classical mechanics and at the same time, the physical system obeys the rule of quantum mechanics. In terms of our approach, one enables to know how quantum process may actually come about. The results are applied to analyze the Landau level. We explain successfully that the orbit of oscillator center for the Landau level is circle.

DOI: 10.12693/APhysPolA.127.761

PACS: 71.70.Di, 73.43.-f, 03.65.Ta, 03.65.Sq

\section{Introduction}

A complete description of the physical state in classical mechanics can be obtained by stating all its coordinates and velocities at a given instant. With these initial data the dynamic equations of classical motion completely determine the behavior of the system at all subsequent times. Different to the classical mechanics the physical state is described by a wave function satisfying the Schrödinger equation [1] in terms of probability interpretation [2]. An equivalent formulating matrix was provided by Born, Jordan and Heisenberg on about the same time [3]. When the location of a microscopic object is observed by experimenters, the probability of finding it in each region depends on the magnitude of its wave function at the time of observation. Therefore it is impossible to determine the coordinates and the corresponding velocities simultaneously.

The wave functions can describe combinations of different states called superpositions. A microscopic object in a superposition of several different locations can occupy several different positions and jumps from one point to another [4]. It appears that the communicate between the superposition is faster than the speed of light. The particles in a well-defined superposition are said to be coherent. The action of observing the quantum superpositions, however, triggers an abrupt change in its wave function, commonly called a collapse according to the Copenhagen interpretation of the quantum mechanics [5]. The measurement problem for the quantum superposition lies at the heart of quantum mechanics and gives rise to many of its paradoxes [6].

\footnotetext{
* corresponding author; e-mail: huipan@umac.mo and zishengwang@yahoo.com
}

For closed physical system in a quantum state of a given energy the wave function is only a property of a statistical ensemble of similarly prepared systems and tells nothing about the time evolution properties of individual physical systems in terms of the probability interpretation. Thus the wave function of the physical system with a constant Hamiltonian in the standard quantum mechanics is time-independent, but it does not mean that individual system does not depend on time. It is clear that the observed world depends on the time $[7,8]$. Therefore, it is necessary to reconcile an observed time-dependence with a time-independent wave function of the universe.

In the domain of dimension of the order of $10^{-13} \mathrm{~cm}$ or less the Copenhagen interpretation to quantum mechanics is totally inadequate as pointed out by Bohm [7]. He tried to describe the seeming quantum randomness in terms of some hidden variables carrying particle internal behavior. The particles actually have fixed positions and momenta at all times but move in a quantum potential in terms of a consequence of the Schrödinger equation. The Bohm theory enables one to understand intuitively and imaginatively where quantum process may actually come about.

Many years later, Bell [9] recasted the Bohm original theory in a very simple and compelling form. He showed that in this case some quantities, which could be measured in certain difficult experiments, would inevitably disagree with the standard quantum predictions.

In the sense, however, the suggestion of hidden variables may be valuable for the statistical ensemble interpretation. In addition the Bohm theory in terms of deterministic particle trajectories [10-12] was originally developed to offer a possible resolution of the interpretative difficulties in the quantum mechanics and serve as a practical tool in various applications [11-17]. 
In this work, differently from the Bohm hidden variable theory, we take the center of mass of physical system as a classical motion but the whole physical systems satisfy still the quantum rule, i.e., the Schrödinger equation, where a time-independent wave function is replaced by a time-dependent one with the observable universe. which may be helpful for one to understand how a quantum process take place. Our approach is applied to describe the motion of an oscillator center for the Landau level.

\section{Particle in magnetic field}

Let us consider a nonrelativistic particle with charge $e$ in an arbitrary external magnetic field described by a vector $\boldsymbol{A}=\boldsymbol{A}(\boldsymbol{r}, t)$ potential with the Hamiltonian,

$$
\mathcal{H}(t)=\frac{1}{2 M}(\hat{\boldsymbol{P}}-e \boldsymbol{A})^{2}=\frac{1}{2 M}(-\mathrm{i} \nabla-e \boldsymbol{A})^{2},
$$

in the units system where $\hbar=1$.

The time-dependent Schrödinger equation is given by

$$
\text { i } \partial_{t} \Psi(\boldsymbol{r}, t)=\mathcal{H}(t) \Psi(\boldsymbol{r}, t) \text {. }
$$

The solution of Eq. (2) can be constructed by the eigenvalues of Hamiltonian (1). The instantaneous eigenequations satisfy

$$
\mathcal{H}(t) \Phi_{n}(\boldsymbol{r}, t)=E_{n}(t) \Phi_{n}(\boldsymbol{r}, t),
$$

where $E_{n}(t)$ are eigenvalues and $\Phi_{n}(\boldsymbol{r}, t)$ are corresponding eigenvactors.

The general solution $\Psi(\boldsymbol{r}, t)$ of the Schrödinger equation can be written as a linear combination in terms of the eigenvectors, i.e.,

$$
\begin{aligned}
& \Psi(\boldsymbol{r}, t)=\sum_{m} c_{m}(t) \Phi_{m}(\boldsymbol{r}, t)= \\
& \sum_{m} a_{m}(t) \exp \left(-\mathrm{i} \int_{0}^{t} E_{m}\left(t^{\prime}\right) \mathrm{d} t^{\prime}\right) \Phi_{m}(\boldsymbol{r}, t),
\end{aligned}
$$

with the initial condition $a_{m}(0)=1$. Inserting Eq. (4) into Eq. (2) and using the adiabatic approximation we obtain

$$
\dot{a}_{n}(t)=-a_{n}(t)\left(\Phi_{n}(\boldsymbol{r}, t), \frac{\mathrm{d}}{\mathrm{d} t} \Phi_{n}(\boldsymbol{r}, t)\right) .
$$

Integrating of Eq. (5) gives

$$
\begin{aligned}
& a_{n}(t)= \\
& a_{n}(0) \exp \left(-\int_{0}^{t}\left(\Phi_{n}\left(\boldsymbol{r}, t^{\prime}\right), \frac{\partial}{\partial t^{\prime}} \Phi_{n}\left(\boldsymbol{r}, t^{\prime}\right)\right) \mathrm{d} t^{\prime}\right),
\end{aligned}
$$

where $a_{n}(0)=1$.

For a normalized eigenstate with the normalization condition $\left(\Phi_{n}(\boldsymbol{r}, t), \Phi_{n}(\boldsymbol{r}, t)\right)=1$ we find

$$
\begin{gathered}
\left(\Phi_{n}(\boldsymbol{r}, t), \frac{\partial}{\partial t} \Phi_{n}(\boldsymbol{r}, t)\right)^{+}= \\
-\left(\Phi_{n}(\boldsymbol{r}, t), \frac{\partial}{\partial t} \Phi_{n}(\boldsymbol{r}, t)\right),
\end{gathered}
$$

which is an imaginary number. Thus

$$
\gamma_{n}^{B}(t)=\mathrm{i} \int_{0}^{t}\left(\Phi_{n}\left(\boldsymbol{r}, t^{\prime}\right), \frac{\partial}{\partial t^{\prime}} \Phi_{n}\left(\boldsymbol{r}, t^{\prime}\right)\right) \mathrm{d} t^{\prime},
$$

is pure phase independently of the dynamic evolution of the system and therefore is called a geometric phase [18]. Inserting Eqs. (8) and (6) into Eq. (4), we find that differently from the constant Hamiltonian system, the state vector $\Psi(\boldsymbol{r}, t)$ include two phases, i.e., the dynamic phase $\gamma_{n}^{d}(t)$ and the geometric phase $\gamma_{n}^{B}(t)$.

The geometric phase (8) associated with cyclic or aperiodical quantum evolution cannot be modified and eliminated by a local gauge transformation $[19,20]$ because of the gauge invariance. Thus the evolving memory of quantum system is kept in terms of such geometric phase.

\section{Stationary state}

Stationary quantum electron states in a constant magnetic field are well-known as quantized Landau states [20, 21]. The Landau levels are essential in the study of quantum Hall effects because their elegant analytical properties enable to construct the fractional states [22-24]. In the following, we consider the behavior of an electron in an external time-independent electromagnetic field described by potential $\boldsymbol{A}=\boldsymbol{A}(\boldsymbol{r})$. One has

$$
\boldsymbol{B}(\boldsymbol{r})=\nabla \times \boldsymbol{A}(\boldsymbol{r}) .
$$

By setting $\Psi(\boldsymbol{r}, t)=\exp (-\mathrm{i} E t) \Phi(\boldsymbol{r})$, the timeindependent Schrödinger equation for the stationary states of the electron is given by

$$
\frac{1}{2 M}(-\mathrm{i} \nabla-e \boldsymbol{A}(\boldsymbol{r}))^{2} \Phi(\boldsymbol{r})=E \Phi(\boldsymbol{r}) .
$$

In order to simplify our calculation without the loss of generalization we consider a simplest magnetic field, i.e., $\boldsymbol{B}=(0,0, B)=$ const. It is obvious that there exists a freedom in the choice of vector potential for a given magnetic field. However, the Hamiltonian is gauge invariant, which means that adding the gradient of a scalar field $\boldsymbol{A}(\boldsymbol{r})$ changes the overall phase of the wave function by an amount corresponding to the scalar field. Physical properties are not influenced by the specific choice of gauge. Under the Landau gauge,

$$
A_{x}=-B y, \quad A_{y}=A_{z}=0,
$$

Eq. (10) is rewritten as

$$
\frac{1}{2 M}\left(\left(\hat{P}_{x}-\frac{e B y}{c}\right)^{2}+\hat{P}_{y}^{2}+\hat{P}_{z}^{2}\right) \Phi(\boldsymbol{r})=E \Phi(\boldsymbol{r})
$$

Note that $\left[H, \hat{P}_{x}\right]=\left[H, \hat{P}_{z}\right]=0$, so it is reasonable to seek for the solution of Eq. (12) in the form

$$
\Phi(\boldsymbol{r})=\exp \left(\mathrm{i}\left(P_{x} x+P_{z} z\right)\right) \chi(y),
$$

where $P_{x}$ and $P_{z}$ are eigenvalues of the operators $\hat{P}_{x}$ and $\hat{P}_{z}$, respectively. Inserting Eq. (13) into Eq. (12), we have

$$
\begin{aligned}
&- \frac{1}{2 M} \chi^{\prime \prime}(y)+\frac{1}{2} M \omega^{2}\left(y-y_{p}\right)^{2} \chi(y)= \\
&\left(E-\frac{P_{z}^{2}}{2 M}\right) \chi(y),
\end{aligned}
$$

where $\omega=\frac{e B}{M c}$ is just a classical frequency of orbital motion of charged particle in magnetic field and $y_{p}=\frac{c P_{x}}{e B}$. Eq. (14) is an equation of harmonic oscillator with an oscillation center $y_{p}$. Thus the solution of Eq. (14) is easy to be obtained as 


$$
\chi_{k}(y)=A_{k} \exp \left(-\frac{\left(y-y_{p}\right)^{2}}{2 l_{B}^{2}}\right) H_{k}\left(\frac{y-y_{p}}{l_{B}}\right),
$$

with the eigenvalues,

$$
E_{k}\left(P_{z}\right)=\frac{1}{2 M} P_{z}^{2}+\left(k+\frac{1}{2}\right) \omega, \quad k \geq 0,
$$

which has an energy band structure characterized by two quantum numbers. Energy levels labeled by $k$ are called Landau levels.

Combining Eq. (13) and (15), the wave vector $\Phi(\boldsymbol{r})$ can be written as

$$
\begin{aligned}
& \Phi_{k}(\boldsymbol{r})=A_{k} \exp \left(\mathrm{i}\left(P_{x} x+P_{z} z\right)-\frac{\left(y-y_{p}\right)^{2}}{2 l_{B}^{2}}\right) \\
& \quad \times H_{k}\left(\frac{y-y_{p}}{l_{B}}\right), \\
& l_{B}=\left(\frac{c}{e B}\right)^{\frac{1}{2}}, \quad A_{k}=\left(\frac{1}{l_{B} \cdot \sqrt{\pi} 2^{k} \cdot k !}\right)^{1 / 2} .
\end{aligned}
$$

The wave function can be factorized as a product of momentum eigenstates in the $x$ - and $z$-directions and harmonic oscillator eigenstates shifted by an amount in the $y$-direction.

It is apparent that the oscillator center in Eq. (16) is spurious, in this sense, because $y_{p}=\frac{c P_{x}}{e B}$ is a constant and not reference point on the $y$-axis. It is, therefore, an indication of breaking the translational symmetry. It is wellknown, however, that the orbits in a magnetic field are circles. Therefore, it is need to look the physical principle for a moving center.

Under the symmetric gauge the vector potential is given by

$$
A_{x}=\frac{1}{2} B y, \quad A_{y}=-\frac{1}{2} B x, \quad A_{z}=0 .
$$

The Schrödinger equation in this gauge is

$\frac{1}{2 M}\left(\left(\hat{P}_{x}-\frac{e B y}{2 c}\right)^{2}+\left(\hat{P}_{y}+\frac{e B x}{2 c}\right)^{2}+\hat{P}_{z}^{2}\right) \Phi=E \Phi$.

Setting

$$
\eta=\frac{x-\mathrm{i} y}{2 l_{B}}, \quad \eta^{*}=\frac{x+\mathrm{i} y}{2 l_{B}},
$$

we define two pairs of creation and annihilation operators as:

$$
a=\frac{1}{\sqrt{2}}\left(\eta+\frac{\partial}{\partial \eta^{*}}\right), \quad a^{+}=\frac{1}{\sqrt{2}}\left(\eta^{*}-\frac{\partial}{\partial \eta}\right),
$$

and

$$
b=\frac{1}{\sqrt{2}}\left(\eta^{*}+\frac{\partial}{\partial \eta}\right), \quad b^{+}=\frac{1}{\sqrt{2}}\left(\eta-\frac{\partial}{\partial \eta^{*}}\right),
$$

with the relations $\left[a, a^{+}\right]=1,\left[b, b^{+}\right]=1$ and $[a, b]=$ $\left[a, b^{+}\right]=0$.

In terms of the creation $a^{+}$and $b^{+}$and annihilation $a$ and $b$ operators, the eigenfunctions in symmetric gauge are

$$
\Phi_{n, m}\left(\eta, \eta^{*}\right)=\mathcal{N}_{n, m}\left(b^{+}\right)^{m}\left(a^{+}\right)^{n} \mathrm{e}^{-\eta^{*} \eta},
$$

where $\mathcal{N}_{n, m}$ is normalized constant.

From Eq. (24), the general eigenfunction can be written as any linear combination $\sum_{m} c_{m} \eta^{m} \Phi_{0}\left(\eta, \eta^{*}\right)$, where $\Phi_{0}\left(\eta, \eta^{*}\right)=\exp \left(-|\eta|^{2}\right)$ is a translation-equivalent state with a different center by picking up an additional (gauge-dependent) phase factors from the vector potential. If the displacement is more than $\sim l_{B}$ the overlap is small with the original state under translating the wave function $\Phi_{0}(\boldsymbol{r})$ with the center $\boldsymbol{r}_{0}=0$ along a straight line to another center $\boldsymbol{r}_{0} \neq 0$.

From Eqs. (16) for the Landau gauge and (24) for the symmetric gauge we see that differently from the time-dependent Hamiltonian system, the stationary state from the Schrödinger Eq. (2) with the form $\Psi(\boldsymbol{r}, t)=$ $\Phi(\boldsymbol{r}) \exp (-\mathrm{i} E t)$ is essentially time-independent because of $|\Psi(\boldsymbol{r}, t)|^{2}=|\Phi(\boldsymbol{r})|^{2}$. At the same time the geometric phase disappears. Therefore it is needed to seek for an observed time-dependence for the physical system. It may be reasonable to express it in terms of the classical motion of oscillator center.

\section{Classical motion of mass center}

The law governing the classical motion of physical systems is the principle of least stationary action $S$, which is a variational principle that when applied to the action of a mechanical system, one can obtain the differential equations of motion of the physical system. The action $S$ is defined by:

$$
S=\int_{t_{1}}^{t_{2}} L\left(x_{c i}, \dot{x}_{c i}, t\right) \mathrm{d} t
$$

where $t$ is time, the dot denotes the time derivative and $L\left(x_{c i}, \dot{x}_{c i}, t\right)$ is a Lagrangian function in which contains all physical information concerning the system and the forces acting on it.

In order to deduce the Hamilton principle, we replace $\frac{\partial L}{\partial \dot{x}_{c i}}$ by $p_{c i}$ in the following:

$$
\begin{aligned}
& d L\left(x_{c i}, \dot{x}_{c i}, t\right)= \\
& \sum_{i}\left(\frac{\partial L}{\partial x_{c i}} \mathrm{~d} x_{c i}+\mathrm{d}\left(p_{c i} \dot{x}_{c i}\right)-\dot{x}_{c i} \mathrm{~d} p_{c i}\right),
\end{aligned}
$$

and

$$
\dot{p}_{c i}=\frac{\mathrm{d} p_{c i}}{\mathrm{~d} t}=\frac{\mathrm{d}\left(\frac{\partial L}{\partial \dot{x}_{c i}}\right)}{\mathrm{d} t}=\frac{\partial L}{\partial x_{c i}} .
$$

Next, we define the Hamiltonian of system as:

$$
\mathcal{H}_{c}\left(p_{c i}, x_{c i}, t\right)=\sum_{i}\left(p_{c i} \dot{x}_{c i}\right)-L .
$$

Using Eqs. (26) and (28), we can write the $\mathrm{d} \mathcal{H}_{c}\left(p_{c i}, x_{c i}, t\right)$ as

$$
\mathrm{d} \mathcal{H}_{c}\left(p_{c i}, x_{c i}, t\right)=-\sum_{i}\left(\frac{\partial L}{\partial x_{c i}} \mathrm{~d} x_{c i}-\dot{x}_{c i} \mathrm{~d} p_{c i}\right) .
$$

On the other hand, $\mathcal{H}_{c}\left(p_{c i}, x_{c i}, t\right)$ is a function of $p_{c i}$ and $x_{c i}$. Thus we have

$$
\mathrm{d} \mathcal{H}_{c}\left(p_{c i}, x_{c i}, t\right)=\sum_{i}\left(\frac{\partial \mathcal{H}_{c}}{\partial p_{c i}} \mathrm{~d} p_{c i}+\frac{\partial \mathcal{H}_{c}}{\partial x_{c i}} \mathrm{~d} x_{c i}\right) .
$$

Comparing Eq. (29) with Eq. (30) and using Eq. (27), we have

$$
\dot{x}_{c i}=\frac{\partial \mathcal{H}_{c}}{\partial p_{c i}}, \dot{p}_{c i}=-\frac{\partial \mathcal{H}_{c}}{\partial x_{c i}}=\frac{\partial L}{\partial x_{c i}},
$$


which is just the Hamilton canonical equation.

Inserting Eq. (28) into Eq. (25) one finds

$S=\int_{t_{1}}^{t_{2}} L\left(x_{c i}, \dot{x}_{c i}, t\right) \mathrm{d} t=\int_{t_{1}}^{t_{2}}\left(\sum_{i}\left(p_{c i} \dot{x}_{c i}\right)-\mathcal{H}_{c}\right) \mathrm{d} t$

Using the Hamilton canonical Eq. (31) we have

$$
\frac{\partial S}{\partial x_{c i}}=\int_{t_{1}}^{t_{2}}\left(-\frac{\partial \mathcal{H}_{c}}{\partial x_{c i}}\right) \mathrm{d} t=\int_{t_{1}}^{t_{2}} \dot{p}_{c i} \mathrm{~d} t=p_{c i},
$$

with the initial condition $p_{c i}(0)=0$.

$\frac{\partial S}{\partial p_{c i}}=\int_{t_{1}}^{t_{2}}\left(\dot{x}_{c i}-\frac{\partial \mathcal{H}_{c}}{\partial p_{c i}}\right) \mathrm{d} t=\int_{t_{1}}^{t_{2}}\left(\dot{x}_{c i}-\dot{x}_{c i}\right) \mathrm{d} t=0$.

Equation (33) can be rewritten as

$$
\nabla_{c} S=\boldsymbol{p}_{c}=M \dot{\boldsymbol{r}}_{c},
$$

which is called as a guidance formula. Equation (35) indicates that a local velocity field can be defined at each point of the system and by integrating in time one can obtain the corresponding classical trajectory.

\section{Motion of center of mass in electromagnetic field}

The Hamiltonian principle is more general than the Newton equation of motion that has broad applicability including electromagnetic fields, the motion of waves, and special relativity.

Using the electromagnetic field Hamiltonian $\mathcal{H}_{c}=$ $\frac{1}{2 M}\left(p_{c}-e \boldsymbol{A}_{c}\right)^{2}+e \varphi\left(\boldsymbol{r}_{c}\right)$ and Hamiltonian Eq. (31) we have

$$
\dot{\boldsymbol{r}}_{c}=\frac{\partial \mathcal{H}_{c}}{\partial \boldsymbol{p}_{c}}=\frac{1}{M}\left(\boldsymbol{p}_{c}-e \boldsymbol{A}_{c}\right)
$$

and

$$
M \ddot{\boldsymbol{r}}_{c}=\dot{\boldsymbol{p}}_{c}-e \dot{\boldsymbol{A}_{c}}=-\frac{\partial \mathcal{H}_{c}}{\partial \boldsymbol{r}_{c}}-e \dot{\boldsymbol{A}_{c}}=e \boldsymbol{E}_{c}+e \boldsymbol{v}_{c} \times \boldsymbol{B}_{c},
$$

where $\boldsymbol{E}_{c}=\nabla_{c} \varphi\left(\boldsymbol{r}_{c}\right)-\frac{\partial \boldsymbol{A}_{c}}{\partial t}$ and $\boldsymbol{B}_{c}=\nabla_{c} \times \boldsymbol{A}_{c}$.

According to Eqs. (27), (33) and (36) the guidance formula is modified as

$$
\nabla_{c} S=\boldsymbol{p}_{c}+e \boldsymbol{A}_{c}=M \boldsymbol{r}_{c}+e \boldsymbol{A}_{c},
$$

in the electromagnetic field.

The solution to Eq. (38) can be written as

$$
S\left(\boldsymbol{r}_{c}, t\right)=M \boldsymbol{r}_{c} \cdot \dot{\boldsymbol{r}}_{c}+e \boldsymbol{r}_{c} \cdot \boldsymbol{A}_{c}+\xi(t),
$$

where $\xi(t)$ will be determined by the energy conservation in the following.

Using Eqs. (38) and (39), we have

$$
\nabla_{c}^{2} S\left(\boldsymbol{r}_{c}(t)\right)=e \nabla_{c} \cdot \boldsymbol{A}_{c},
$$

and

$$
\frac{\partial S}{\partial t}=M \dot{\boldsymbol{r}_{c}^{2}}+M \boldsymbol{r}_{c} \cdot \ddot{\boldsymbol{r}}_{c}+e \dot{\boldsymbol{r}_{c}} \cdot \boldsymbol{A}_{c}+e \boldsymbol{r}_{c} \cdot \dot{\boldsymbol{A}_{c}}+\frac{\mathrm{d} \xi}{\mathrm{d} t} .
$$

\section{Wave function in classical mass center}

The wave functions (16) and (24) of stationary state with a given energy from the Schrödinger equation with the form $\Psi(\boldsymbol{r}, t)=\Phi(\boldsymbol{r}) \exp (-\mathrm{i} E t)$ does not mean that individual system does not depend on time [25, 26], while the whole phase factor $\exp (-\mathrm{i} E t)$ may be dropped off in the quantum calculation. Especially, the probability is $|\Psi(\boldsymbol{r}, t)|^{2}=|\Phi(\boldsymbol{r})|^{2}$ does not depend on evolving time. Thus Eqs. (16) and (24) do tell nothing about the time evolution properties of individual physical systems.

In order to seek for the time evolution properties of physical $s$ stem, we rephrase the Schrödinger Eq. (2) as invariant under the acting transformation

$$
\Psi\left(\boldsymbol{r}-\boldsymbol{r}_{c}(t)\right)=\mathrm{e}^{\mathrm{i} S\left(\boldsymbol{r}_{c}(t)\right)} \Phi\left(\boldsymbol{r}-\boldsymbol{r}_{c}(t)\right),
$$

where $S\left(\boldsymbol{r}_{c}(t)\right)$ is the classical action satisfying Eq. (38) and $\boldsymbol{r}_{c}(t)$ is a center coordinate of physical system.

A located scatter center of an effective two-dimensional potential is important for the integer quantum Hall effect as pointed out by Ando, Matsumoto and Uemura [27] because the relation between the Hall conductivities $\sigma_{X Y}$ and $\sigma_{X X}$ can be deduced. In the following, therefore, we separate the vector potential into two parts, i.e.:

$$
\boldsymbol{A}(\boldsymbol{r})=\boldsymbol{A}_{R}+\boldsymbol{A}_{c}\left(\boldsymbol{r}_{c}\right)
$$

with $\boldsymbol{A}_{c}=\boldsymbol{A}_{c}\left(\boldsymbol{r}_{c}\right)$ and $\boldsymbol{A}_{R}=\boldsymbol{A}(\boldsymbol{r})-\boldsymbol{A}_{c}\left(\boldsymbol{r}_{c}\right)$. Thus we have

$$
\begin{aligned}
& \frac{1}{2 M}(\boldsymbol{P}-e \boldsymbol{A}(\boldsymbol{r}))^{2} \Psi\left(\boldsymbol{r}-\boldsymbol{r}_{c}(t)\right)= \\
& \quad \frac{1}{2 M}\left(\left(-i \nabla_{R}-e \boldsymbol{A}_{R}\right)-e \boldsymbol{A}_{c}\right)^{2} \Psi\left(\boldsymbol{r}-\boldsymbol{r}_{c}(t)\right)= \\
& \quad \frac{1}{2 M} \mathrm{e}^{\mathrm{i} S}\left\{\left(\nabla_{c} S-e \boldsymbol{A}_{c}\right)^{2}+\left(-\mathrm{i} \nabla_{R}-e \boldsymbol{A}_{R}\right)^{2}\right. \\
& \quad+2\left(\nabla_{c} S-e \boldsymbol{A}_{c}\right) \cdot\left(-\mathrm{i} \nabla_{R}-e \boldsymbol{A}_{R}\right) \\
& \left.+\left(-\mathrm{i} \nabla_{c}^{2} S+\mathrm{i} e \nabla_{c} \cdot \boldsymbol{A}_{c}\right)\right\} \Phi\left(\boldsymbol{r}-\boldsymbol{r}_{c}(t)\right),
\end{aligned}
$$

where $\boldsymbol{r}=\boldsymbol{r}_{c}+\boldsymbol{R}, \nabla_{c}=\frac{\partial}{\partial \boldsymbol{r}_{c}}$ and $\nabla_{R}=\frac{\partial}{\partial \boldsymbol{R}}$. On the other hand, one finds

$$
\begin{gathered}
\mathrm{i} \frac{\partial \Psi}{\partial t}=\mathrm{e}^{\mathrm{i} S}\left(-\frac{\partial S}{\partial t}+\mathrm{i} \frac{\partial}{\partial t}\right) \Phi\left(\boldsymbol{r}-\boldsymbol{r}_{c}(t)\right)= \\
\mathrm{e}^{\mathrm{i} S}\left(-\frac{\partial S}{\partial t}-\mathrm{i} \dot{\boldsymbol{r}}_{c} \cdot \nabla_{R}\right) \Phi\left(\boldsymbol{r}-\boldsymbol{r}_{c}(t)\right) .
\end{gathered}
$$

Inserting Eqs. (44) and (45) into Eq. (2) and using Eqs. (39)-(41), we find

$$
\begin{gathered}
{\left[-\frac{\mathrm{d} \xi}{\mathrm{d} t}-\frac{3}{2} M \dot{\boldsymbol{r}_{c}^{2}}-M \boldsymbol{r}_{c} \cdot \ddot{\boldsymbol{r}}_{c}-e \dot{\boldsymbol{r}_{c}} \cdot \boldsymbol{A}_{c}-e \boldsymbol{r}_{c} \cdot \dot{\boldsymbol{A}}_{c}\right.} \\
\left.-\frac{1}{2 M}\left(-\mathrm{i} \nabla_{R}-e \boldsymbol{A}(\boldsymbol{R})\right)^{2}+e \dot{\boldsymbol{r}_{c}} \cdot \boldsymbol{A}_{R}\right] \Phi=0 .
\end{gathered}
$$

Determining the function $\xi$ by

$$
\begin{aligned}
& \frac{\mathrm{d} \xi}{\mathrm{d} t}+\frac{3}{2} M \dot{\boldsymbol{r}_{c}^{2}}+M \boldsymbol{r}_{c} \cdot \ddot{\boldsymbol{r}}_{c}+e \dot{\boldsymbol{r}_{c}} \cdot \boldsymbol{A}_{c} \\
& \quad+e \boldsymbol{r}_{c} \cdot \dot{\boldsymbol{A}_{c}}-e \dot{\boldsymbol{r}_{c}} \cdot \boldsymbol{A}_{R}=-E,
\end{aligned}
$$

we have

$$
\frac{1}{2 M}\left(-\mathrm{i} \nabla_{R}-e \boldsymbol{A}_{R}\right)^{2} \Phi\left(\boldsymbol{r}-\boldsymbol{r}_{c}(t)\right)=E \Phi\left(\boldsymbol{r}-\boldsymbol{r}_{c}(t)\right),
$$

which has the same form as Eq. (10). However, Eq. (48) includes the motion of center of mass as the know how a quantum process comes about.

Similarly to Eq. (12), under the Landau gauge with $A_{R_{x}}=-B\left(y-y_{0}(t)\right)$ and $A_{R_{y}}=A_{R_{z}}=0$, the solution of Eq. (48) is given by 


$$
\begin{aligned}
& \Phi_{k}\left(\boldsymbol{r}-\boldsymbol{r}_{c}(t)\right)= \\
& A_{k} \exp \left(\mathrm{i}\left(P_{x}\left(x-x_{c}\right)+P_{z}\left(z-z_{c}\right)\right)-\frac{\left(y-y_{c}-y_{p}\right)^{2}}{2 l_{B}^{2}}\right) \\
& \times H_{k}\left(\frac{y-y_{c}-y_{p}}{l_{B}}\right),
\end{aligned}
$$

where $A_{k}$ is normalization factor.

Under the symmetric gauge with $A_{R_{x}}=\frac{1}{2} B\left(y-y_{c}(t)\right)$, $A_{R_{y}}=-\frac{1}{2} B\left(x-x_{c}(t)\right)$ and $A_{R_{z}}=0$, similarly to Eq. (20), the solution of Eq. (49) is expressed as

$$
\Phi_{n, m}\left(\boldsymbol{r}-\boldsymbol{r}_{c}(t)\right)=\mathcal{N}_{n, m}\left(b^{+}\right)^{m}\left(a^{+}\right)^{n} \mathrm{e}^{-\left|\eta-\eta_{c}(t)\right|^{2}},
$$

where $\mathcal{N}_{n, m}$ is a normalization constant.

In constant magnetic field $\boldsymbol{A}=\boldsymbol{r} \times \boldsymbol{B}$, the determining function $\xi$ can be expressed by

$$
\frac{\mathrm{d} \xi}{\mathrm{d} t}+\frac{3}{2} M \dot{\boldsymbol{r}_{c}^{2}}+M \boldsymbol{r}_{c} \cdot \ddot{\boldsymbol{r}_{c}}=-E,
$$

which is independent of the potential $\boldsymbol{A}$.

Integrating to Eq. (49), we have

$$
\begin{gathered}
\xi(t)=-E t-\frac{3 M}{2} \int_{0}^{t} \dot{\boldsymbol{r}_{c}^{2}}\left(t^{\prime}\right) \mathrm{d} t^{\prime} \\
-M \int_{0}^{t} \boldsymbol{r}_{c}\left(t^{\prime}\right) \cdot \ddot{\boldsymbol{r}}_{c}\left(t^{\prime}\right) \mathrm{d} t^{\prime} .
\end{gathered}
$$

The center of mass coordinate can be obtained by the Lorentz Eq. (37), we find

$$
\begin{aligned}
& x_{c}(t)=\frac{v_{0 x}}{\omega} \sin \omega t+\frac{v_{0 y}}{\omega}(1-\cos \omega t)+x_{c}(0), \\
& y_{c}(t)=-\frac{v_{0 x}}{\omega}(1-\cos \omega t)+\frac{v_{0 y}}{\omega} \sin \omega t+y_{c}(0), \\
& z_{c}(t)=0,
\end{aligned}
$$

where $x_{c}(0), y_{c}(0), v_{0 x}$ and $v_{0 y}$ are constants determined by the initial conditions. Equations (53) $-(55)$ are indeed cyclic motion with the cyclicity $T=2 \pi / \omega$. Thus we successfully explain that the orbit of oscillator center for the Landau level is circle.

From Eqs. (2) and (49), the state vectors $\Phi(\boldsymbol{r}-$ $\left.\boldsymbol{r}_{c}(t)\right)=\exp \left(i S\left(\boldsymbol{r}_{c}(t)\right)\right) \Psi\left(\boldsymbol{r}-\boldsymbol{r}_{c}(t)\right)$ satisfies exactly the Schrödinger equation. However, it points in very nice fashion the important role played by a dynamic phase $S\left(\boldsymbol{r}_{c}(t)\right)$ determining the evolving track of motion in quantum mechanics. Especially, the time-dependent probability amplitude $\left|\Psi\left(\boldsymbol{r}-\boldsymbol{r}_{c}(t)\right)\right|^{2}$ makes one to understand intuitively and imaginatively how quantum process may actually come about just like the Bohm theory. In principle our results are more general, reaching beyond the standard quantum mechanics in which the time-dependent wave functions are totally neglected.

Under the situation of $\nabla_{c} \times \boldsymbol{A}_{c}=\boldsymbol{B}_{c}=0$ with $\boldsymbol{A}_{c} \neq 0$ we see from Eqs. (41), (47) and (52) that the additional phase $S\left(\boldsymbol{r}_{c}\right)$ in Eq. (42) would not disappear. The result can successfully explain the Aharonov-Bohm effect [27] in which predicts the production of a relative phase shift between two electron beams enclosing a magnetic flux even if they do not touch the magnetic flux.

\section{Discussions and conclusions}

In summary, the proposed approach gives new picture of quantum mechanics, leading to more natural scenario than the standard one based on abstract and subjective concepts, where the quantum state vector obeying the Schrödinger equation includes the center motion of physical system in terms of the classical mechanics. The results show that the wave functions are time-dependent as well as the probability amplitudes, which is just needed for the observable.

In conventional quantum mechanics, the motion track of concepts is lost. The state vectors are only a property of a statistical ensemble of similarly prepared systems and tells nothing about the time evolution properties of individual physical systems obeying the probability interpretation. In terms of the Ehrenfest theorem, the time derivative of the expectation value for a quantum mechanical observable is connected to classical mechanics. Differently from the Ehrenfest theorem [28], we seek for direct connection of the classical theory with quantum mechanics by introducing the center motion of quantum wave packet satisfied the classical rule. Our approach may offer an appropriate tool to overcome such an intermediate gap. The results are important to better learn and understand the physics behind microscopic particle. Thus the trajectory concept is consistently introduced into the quantum mechanics scenarios. It is necessary to emphasize that our approach is different from the Bohm theory and the hidden variables are not needed.

Such a unified description of both the standard quantum theory for microscopic objects and the classical behavior for macroscopic objects is similar to the GRW theory [29]. In the GRW theory a modified quantum dynamics with the trajectory concept was constructed by introducing a stochastic term corresponding to a localization in the dynamical equation and the wave-packet reduction with definite final pointer position was deduced. Thus the occurrence of linear superposition of quantum states localized in far-away spatial regions are naturally suppressed inducing an evolution consisted with classical mechanics. In our approach, such stochastic variable is not necessary in the wave-packet dynamics.

These results were applied to analyze the Landau level. We find that quantum electron in constant magnetic field has stationary state. Wave function with additional phase factor is satisfying a classical guidance formula in quantum mechanics. The orbit of oscillator center determined by the Lorentz equation is naturally a circle.

\section{Acknowledgments}

This work is supported by the Natural Science Foundation of China under Grant No. 11365012, the Natural Science Foundation of Jiangxi Province, China under Grant No. 20132BAB202008, and the Foundation of Science and Technology of Education Office of Jiangxi Province under Grant No. GJJ13235. 
Hui Pan thanks the supports of the Science and Technology Development Fund from Macao SAR (FDCT068/2014/A2 and FDCT 132/2014/A3), and MultiYear Research Grants (MYRG2014-00159-FST and MYRG2015-0015- FST) and Start-up Research Grant (SRG-2013- 00033-FST) from Research and Development Office at University of Macau.

\section{References}

[1] E. Schrödinger, Ann. Phys. 384, 361 (1926); ibid., 734 (1926).

[2] M. Born, Z. Phys. 38, 803 (1926).

[3] W. Heisenberg, Z. Phys. 33, 879 (1925); M. Born, P. Jordan, Z. Phys. 34, 858 (1925); M. Born, W. Heisenberg, P. Jordan, Z. Phys. 35, 557 (1926).

[4] E. Schrödinger, Proc. Cambr. Philos. Soc. 31, 555 (1935); ibid. 32, 446 (1936).

[5] N. Bohr, Essays 1958-1962 on Atomic Physics and Human Knowledge, Ox Bow Press, Woodbridge Connecticut Margenau 1963, p. 56.

[6] Z.S. Wang, R.S. Wu, Int. J. Theor. Phys. 48, 1859 (2009).

[7] D. Bohm, Phys. Rev. 85, 180 (1952); ibid., 166 (1952); D. Bohm, J.P. Vigier, Phys. Rev. 96, 208 (1954).

[8] A.R. Plastino, M. Casas, A. Plastino, Phys. Lett. A 281, 297 (2001).

[9] J.S. Bell, Physica 1, 195 (1964).

[10] Z.S. Wang, L.C. Kwek, C.H. Lai, C.H. Oh, Phys. Lett. A 359, 608 (2006).

[11] D. Bohm, B.J. Hiley, The Undivided Universe, Routledge, London 1993.
[12] R.E. Wyatt, Quantum Dynamics with Trajectories, Springer, New York 2005.

[13] D. Dürr, S. Teufel, Bohmian Mechanics, Springer, Berlin 2009.

[14] C.L. Lopreore, R.E. Wyatt, Phys. Rev. Lett. 82, 5190 (1999).

[15] L.R. Pettey, R.E. Wyatt, Int. J. Quantum Chem. 107, 1566 (2007).

[16] S. Hamada, H. Sekino, Int. J. Quantum Chem. 111, 1480 (2011).

[17] Z.S. Wang, Chunfeng Wu, Xun-Li Feng, L.C. Kwek, C.H. Lai, C.H. Oh, Phys. Lett. A 372, 775 (2008)

[18] M.V. Berry, Proc. R. Soc. A 392, 45 (1984).

[19] Z.S. Wang, Qian Liu, Phys. Lett. A 377, 3272 (2013).

[20] J. Samuel, R. Bhandari, Phys. Rev. Lett. 60, 2339 (1988).

[21] L.D. Landau, E.M. Lifshitz, Quantum Mechanics: Non-relativistic Theory, Butterworth-Heinemann, London 1981.

[22] C. Cohen-Tannoudji, B. Diu, F. Laloë, Quantum Mechanics, Wiley-Interscience, New York 2006.

[23] D.-J. Thouless, M. Kohmoto, M.-P. Nightingale, M. den Nijs, Phys. Rev. Lett. 49, 405 (1982).

[24] M. Kohmoto, Ann. Phys. 160, 343 (1985).

[25] F.D.M. Haldane, Phys. Rev. Lett. 61, 2015 (1988).

[26] A. Tsuneya, Y. Matsumoto, Y. Uemura, J. Phys. Soc. Jpn. 39, 279 (1975)

[27] Y. Aharonov, D. Bohm, Phys. Rev. 115, 485 (1959).

[28] P. Ehrenfest, Z. Phys. 45, 455 (1927).

[29] G.C. Ghirardi, A. Rimini, T. Weber, Phys. Rev. D 34, 470 (1986). 